Chronische myeloische Leukämie

\section{Absetzen von Imatinib ist möglich}

Die Therapie mit Imatinib kann bei Patienten mit chronischer myeloischer Leukämie (CML), bei denen über mindestens zwei Jahre eine nicht detektierbare Resterkrankung (UMRD) vorliegt, sicher unterbrochen werden. Dies bestätigen die endgültigen Ergebnisse der prospektiven französischen Stopp-Imatinib-Studie (STIM1).

In der Studie wurde die Imatinib-Therapie bei 100 Patienten mit CML, die über $\geq 2$ Jahre eine UMRD aufwiesen, abgesetzt. Bei Auftreten eines molekularen Rezidivs sollte die Therapie sofort wieder aufgenommen werden.

Das mediane molekulare Follow-up nach dem Abbruch der Imatinib-Therapie betrug 77 Monate. 61 Patienten verloren den UMRD-Status nach median 2,5 Monaten und ein Patient verstarb ohne Verlust der UMRD nach zehn Monaten. Ein molekulares Rezidiv trat meist innerhalb von sechs Monaten (im Median 2,5 Monaten) nach Therapieunterbrechung auf.
Die Rate für das molekulare rückfallfreie Überleben betrug $43 \%$ nach sechs Monaten und $38 \%$ nach 60 Monaten. Die Imatinib-Therapie wurde bei 57 von 61 Patienten mit molekularem Rezidiv wieder aufgenommen, und 55 der 57 Patienten erzielten eine zweite UMRD nach median vier Monaten. Keiner der Patienten erlebte eine Progression der CML. Beim letzten Follow-up-Termin lebten noch 57 Patienten; 43 Patienten (70\%) erhielten einen Tyrosinkinaseinhibitor (TKI), 14 standen nicht unter TKI, neun davon befanden sich in Therapiepause.

In Analysen der Ausgangscharakteristika der Studienpopulation wurden der Sokal-Score und die Dauer der ImatinibTherapie als signifikante unabhängige Risikofaktoren identifiziert für ein molekulares Rezidiv. Brigitte Schalhorn

Etienne $\mathrm{G}$ et al. Long-term follow-up of the french stop imatinib (STIM1) study in patients with chronic myeloid leukemia. J Clin Oncol. 2016;35(3):298-305.

\section{kurz notiert}

Forschungsstandort Deutschland

Sensor für Krebsgen entwickelt +++ Für TP53, das wichtigste humane Tumorsuppressorgen, haben Forscher der TU Dresden nun einen molekularen Sensor entwickelt, der anzeigt, ob TP53 in seiner Funktion gestört ist. Ist TP53 defekt, führt dies zur Aktivierung des Sensors und die Zerstörung der Zelle wird eingeleitet [Mircetic J et al. Nat Commun. 2017;8(1):1463]. red.

Neue Tumortherapien für Kinder +++ Das Forschungskonsortium ITCC-P4 setzt sich zum Ziel, die Entwicklung neuer Medikamente für krebskranke Kinder zu beschleunigen und neue Biomarker zu entwickeln, um die Medikamente möglichst passgenau einsetzen zu können (https://www.itccp4.eu/), heißt es einer Mitteilung des Deutschen Krebsforschungszentrums (DKFZ). Dazu sollen für zehn verschiedene Krebsarten bei Kindern, die bisher oftmals nur unzureichend behandelt werden können, patientenindividuelle präklinische Modelle entwickelt werden. Die Wissenschaftler gehen davon aus, dass sie mit den patientenindividuellen Tumormodellen aussagekräftigere Ergebnisse zur Vorbereitung klinischer Studien erzielen, als es momentan mit den bisher üblichen Tests an Krebszellen in der Kulturschale oder an genetisch gleichförmigen Mausstämmen möglich ist. red.

genüber $51 \%$ und $50 \%$ unter den Monotherapien mit Chlorambucil bzw. Rituximab.

Das PFS stieg durch die Kombination signifikant an (HR 0,62; p = 0,0119). Während hier der Medianwert noch nicht erreicht war, lag er unter Chlorambucil bei 8,3 Monaten und unter Rituximab bei 6,9 Monaten. Nach fünf Jahren lebten $72 \%$ der kombiniert Behandelten progressionsfrei, unter Chlorambucil bzw. Rituximab waren es $59 \%$ bzw. $57 \%$.

Im Gesamtüberleben wurden jedoch keine Unterschiede nachgewiesen, die 5-Jahres-Überlebensrate betrug unter der Kombination $89 \%$ gegenüber den Monotherapien mit $89 \%$ (Chlorambucil) und 92\% (Rituximab).

Die Therapien wurden gut toleriert, unerwartete Toxizitäten wurden nicht berichtet.

Brigitte Schalhorn

Zucca E et al. Final results of the IELSG-19 randomized trial of mucosa-associated lymphoid tissue lymphoma: Improved event-free and progression-free survival with rituximab plus chlorambucil versus either chlorambucil or rituximab monotherapy. J Clin Oncol. 2017;35(17):1905-12. 